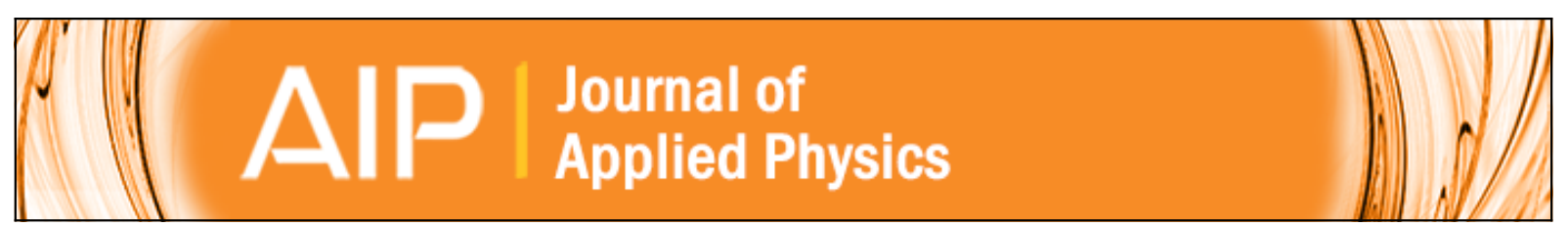

First principle analyses of direct bandgap solar cells with absorbing substrates versus mirrors

Alexander P. Kirk and Wiley P. Kirk

Citation: Journal of Applied Physics 114, 174507 (2013); doi: 10.1063/1.4829459

View online: http://dx.doi.org/10.1063/1.4829459

View Table of Contents: http://scitation.aip.org/content/aip/journal/jap/114/17?ver=pdfcov

Published by the AIP Publishing

$\underset{\substack{\text { Publoning } \\ \text { Alp }}}{A}$ Re-register for Table of Content Alerts

Create a profile.

Sign up today! 


\title{
First principle analyses of direct bandgap solar cells with absorbing substrates versus mirrors
}

\author{
Alexander P. Kirk ${ }^{1}$ and Wiley P. Kirk ${ }^{2}$ \\ ${ }^{1}$ School of Electrical, Computer and Energy Engineering, Arizona State University, Tempe, \\ Arizona 85287, USA \\ ${ }^{2}$ Department of Materials Science and Engineering, University of Texas at Arlington, Arlington, \\ Texas 76019, USA
}

(Received 19 September 2013; accepted 22 October 2013; published online 7 November 2013)

\begin{abstract}
Direct bandgap InP, GaAs, CdTe, and $\mathrm{Ga}_{0.5} \mathrm{In}_{0.5} \mathrm{P}$ solar cells containing backside mirrors as well as parasitically absorbing substrates are analyzed for their limiting open circuit voltage and power conversion efficiency with comparison to record solar cells. From the principle of detailed balance, it is shown quantitatively that mirror solar cells have greater voltage and power conversion efficiency than their substrate counterparts. Next, the radiative recombination coefficient and maximum radiative lifetime of GaAs mirror and substrate solar cells are calculated and compared to the nonradiative Auger and Shockley-Read-Hall (SRH) lifetimes. Mirror solar cells have greater radiative lifetime than their substrate variants. Auger lifetime exceeds radiative lifetime for both substrate and mirror cells while SRH lifetime may be less or greater than radiative lifetime depending on trap concentration and capture cross section. Finally, the change in free energy of the photogenerated carriers is analyzed in a comparison between InP, GaAs, CdTe, and $\operatorname{Ga}_{0.5} \operatorname{In}_{0.5} \mathrm{P}$ mirror and substrate solar cells in order to characterize the relationship between solar photon quality and free energy management in solar cells with differing bandgaps. Wider bandgap visible threshold $\mathrm{Ga}_{0.5} \mathrm{In}_{0.5} \mathrm{P}$ solar cells make better use of the available change in free energy of the photogenerated charge carriers, even when normalized to the bandgap energy, than narrower bandgap near-IR threshold InP, GaAs, and CdTe solar cells. (C) 2013 AIP Publishing LLC. [http://dx.doi.org/10.1063/1.4829459]
\end{abstract}

\section{INTRODUCTION}

Solar cells are typically designed with one of three configurations: (1) on an absorbing substrate such as a semiconductor wafer, (2) with an evaporated backside mirror such as $\mathrm{Au}$ or Ag, or (3) with two air exposed surfaces top and bottom in what is commonly known as a bifacial configuration. Option 3 exists in indirect bandgap Si wafer-based solar cells such as Panasonic's HIT $^{\circledR}$ Double bifacial cells. In this paper, the goal is to analyze and compare options 1 and 2 for direct bandgap InP, GaAs, CdTe, and $\mathrm{Ga}_{0.5} \mathrm{In}_{0.5} \mathrm{P}$ compound semiconductor solar cells by considering important solar cell parameters including open circuit voltage, power conversion efficiency, radiative lifetime, and nonradiative Auger lifetime as well as nonradiative Shockley-Read-Hall (SRH) lifetime. All of the calculations presented here are made directly from first principles thus avoiding fitting coefficients or ideality factors that can inadvertently mask the relevant physics. Calculated data, where directly relevant, are compared to published performance data for world record solar cells as taken from the 42 nd solar cell efficiency tables. ${ }^{1}$

\section{OPEN CIRCUIT VOLTAGE}

The principle of detailed balance ${ }^{2}$ was used to calculate the open circuit voltage $V_{o c}$ for solar cells under the AM1.5G solar spectrum (ASTM G173-03) at $298 \mathrm{~K}$. ${ }^{1}$ These calculations were made for perfectly reflecting mirror solar cells as well as for parasitically absorbing substrate solar cells. Assumptions are that photons with energy $h v \geq E_{g}$ are absorbed (where $E_{g}$ is bandgap) while photons with energy $h v<E_{g}$ are not absorbed, nonradiative recombination is assumed to be absent, steadystate quasi-Fermi level separation is constant, there is no parasitic $I^{2} R$ power loss, each absorbed photon generates one electron and hole, and the Boltzmann approximation applies.

If the solar cell has a perfectly reflecting backside mirror, then the expression ${ }^{3}$ for the detailed balance-limiting $V_{o c}$ is given by

$$
V_{o c}=E_{g} e^{-1}-k_{B} T e^{-1} \ln \left(2 \pi e E_{g}^{2} k_{B} T h^{-3} c^{-2} J_{p h}^{-1}\right),
$$

where $e$ is electronic charge, $k_{B}$ is Boltzmann's constant, $T$ is cell temperature, $h$ is Planck's constant, $c$ is speed of light, and $J_{p h}$ is photogenerated current density. The spectral photon flux for photons with energy $h v \geq E_{g}$ is integrated (by using Origin $^{\circledR}$ v8 graphing software and trapezoidal rule numerical integration) and then multiplied by the electronic charge in order to calculate $J_{p h}$. The bandgap values used here are $1.34 \mathrm{eV}(\mathrm{InP}), 1.42 \mathrm{eV}$ (GaAs), $1.50 \mathrm{eV}$ (CdTe), and $1.81 \mathrm{eV}$ (Ref. 4) $\left(\mathrm{Ga}_{0.5} \mathrm{In}_{0.5} \mathrm{P}\right)$.

If the solar cell is fabricated instead on a parasitically absorbing substrate, then the expression for the detailed balance-limiting $V_{o c}$ is now given by

$$
V_{o c}=E_{g} e^{-1}-k_{B} T e^{-1} \ln \left[2 \pi\left(1+n_{r}^{2}\right) e E_{g}^{2} k_{B} T h^{-3} c^{-2} J_{p h}^{-1}\right],
$$

where $n_{r}$ is the index of refraction of the parasitically absorbing substrate. The term "parasitically absorbing" here means 
that when the substrate absorbs photons it neither participates actively in the photovoltaic process nor appreciably radiates its own luminescent photons that could be available for absorption in the active region of the solar cell that is located above. For example, the substrate could be comprised of a semi-insulating wafer whereby recombination tends to be dominantly nonradiative. It is assumed that the parasitically absorbing substrates are comprised of InP for the InP solar cells, GaAs for the GaAs solar cells, CdTe for the CdTe solar cells, and GaAs for the lattice-matched $\mathrm{Ga}_{0.5} \mathrm{In}_{0.5} \mathrm{P}$ solar cells. The values of $n_{r}$ corresponding to the bandgap energy of the individual solar cells are 3.385 (InP), 3.653 (GaAs), 3.037 (CdTe), and 3.784 ( $n_{r}$ value for GaAs substrate corresponding to $1.81 \mathrm{eV}$ photons emitted by $\left.\mathrm{Ga}_{0.5} \operatorname{In}_{0.5} \mathrm{P}\right){ }^{5}$

A comparison of $V_{o c}$ for world record solar cells and detailed balance-limiting mirror and substrate cells is shown in Fig. 1. As governed by the principle of detailed balance, mirror solar cells have greater limiting $V_{o c}$ than parasitically absorbing substrate solar cells. ${ }^{3}$ A larger value of $n_{r}$ leads to greater reduction in $V_{o c}$ (Eq. (2)). The best performing cells that have actually been fabricated are indeed those that have high quality backside mirrors and epitaxial single crystal active regions as is the case for the record GaAs and $\mathrm{Ga}_{0.5} \mathrm{In}_{0.5} \mathrm{P}$ cells. The world record $\mathrm{GaAs}$ and $\mathrm{Ga}_{0.5} \mathrm{In}_{0.5} \mathrm{P}$ mirror cells have already equaled or exceeded the limiting voltage (but not efficiency as shown later in Table I) of their detailed balance-limiting substrate cell counterparts. The record InP cell is a single crystal device on an InP wafer and the record CdTe cell is a polycrystalline device. ${ }^{1}$

\section{POWER CONVERSION EFFICIENCY}

When $d(J V) / d V=0$, the maximum power point voltage $V_{m}$ is given by

$$
V_{m}=V_{o c}-k_{B} T e^{-1} \ln \left(1+e V_{m} / k_{B} T\right) .
$$

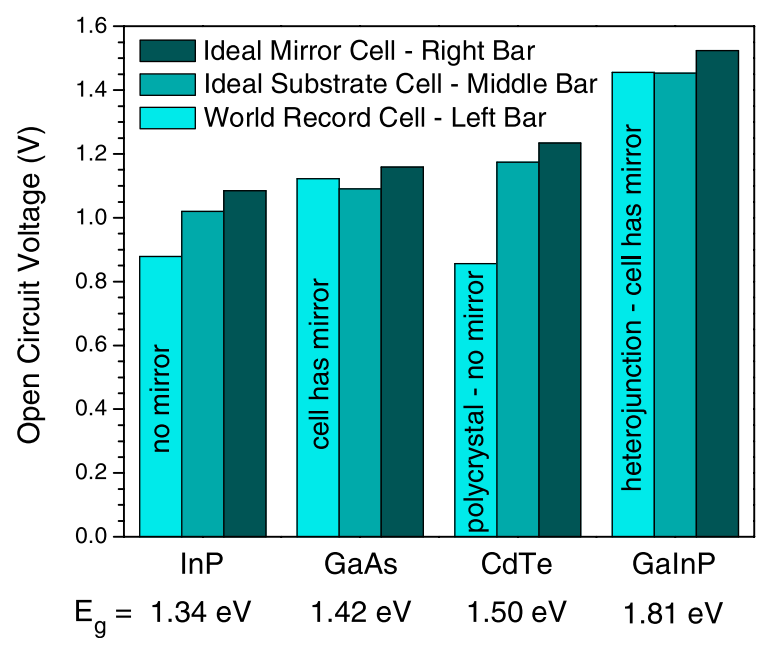

FIG. 1. Comparison of $V_{o c}$ for world record solar cells vs. detailed balancelimiting substrate and mirror solar cells operating under the AM1.5G1× spectrum at $298 \mathrm{~K}$. Note that the term "ideal" here refers to detailed balancelimiting mirror (substrate) cells. Without specific device fabrication details (in Ref. 1) the world record CdTe solar cell is assumed not to have a mirror.
The previous equation requires numerical iteration to solve. However, as an approximation, Eq. (3) may instead be rewritten as

$$
V_{m} \approx V_{o c}-k_{B} T e^{-1} \ln \left(1+e V_{o c} / k_{B} T\right) .
$$

The error in determining $V_{m}$ using Eq. (4) instead of Eq. (3), with a GaAs solar cell as an example, is only $0.2 \%$ which makes Eq. (4) a suitable approximation as used in this paper.

The maximum power point current density $J_{m}$ is given by

$$
J_{m}=J_{p h} /\left(1+k_{B} T / e V_{m}\right)
$$

The power conversion efficiency $\eta$ is then given by

$$
\eta=P_{\text {out }} / P_{\text {in }}
$$

where $P_{\text {out }}=J_{m} V_{m}$ and $P_{\text {in }}=0.1 \mathrm{~W} \mathrm{~cm}{ }^{-2}$ for the AM1.5G spectrum at $1 \times$ (one Sun illumination). World record efficiency $^{1}$ and detailed balance-limiting efficiency for the solar cells are shown in Table I.

From Table I, it is worth noting that the reported world record GaAs and $\mathrm{Ga}_{0.5} \mathrm{In}_{0.5} \mathrm{P}$ solar cells which benefit from high quality mirrors have now achieved measured $V_{o c}$ values that exceed the theoretical (detailed balance-limiting) $V_{o c}$ of their substrate cell counterparts. However, these record setting GaAs and $\mathrm{Ga}_{0.5} \mathrm{In}_{0.5} \mathrm{P}$ mirror cells have not yet exceeded the theoretical (detailed balance-limiting) power conversion efficiency of their substrate cell counterparts, despite greater $V_{o c}$, because these record setting mirror cells have lower values of measured current density (not shown here) than the ideal substrate cells, and power conversion efficiency is a function of both voltage and current density.

\section{RADIATIVE RECOMBINATION COEFFICIENT}

In this section, the radiative recombination coefficient of GaAs mirror vs. substrate solar cells will be determined. The principle of detailed balance may be used to calculate the radiative recombination coefficient $B$ for mirror and substrate solar cells. If the solar cell has a perfectly reflecting backside mirror, then the expression ${ }^{6}$ for the radiative recombination coefficient is given by

$$
B=2 \pi n_{i}^{-2} h^{-3} c^{-2} \int_{E_{g}}^{\infty} E^{2} \alpha(E) \exp \left(-E / k_{B} T\right) d E,
$$

where $E$ represents the photon energy, $\alpha(E)$ is the absorption coefficient, and $n_{i}$ is the intrinsic carrier concentration ${ }^{7}$ given by

$$
n_{i}=\sqrt{N_{c} N_{v}} \exp \left(-E_{g} / 2 k_{B} T\right),
$$

where $N_{c(v)}$ is the effective density of conduction(valence) band states. From measured values of electron, light hole, and heavy hole effective masses at low temperature $(\sim 4 \mathrm{~K})$, the extrapolated estimate of $N_{c}$ and $N_{v}$ for GaAs at $300 \mathrm{~K}$ are $4.21 \times 10^{17} \mathrm{~cm}^{-3}$ (with correction for the non-parabolic 
TABLE I. Cell efficiency (and $V_{o c}$ ); AM1.5G $1 \times$ spectrum; $298 \mathrm{~K}$. Note the bandgap values in columns increase from left to right.

\begin{tabular}{lcccc}
\hline \hline & $\mathrm{InP}$ & $\mathrm{GaAs}$ & $\mathrm{CdTe}$ & $\mathrm{GaInP}$ \\
Bandgap & $1.34 \mathrm{eV}$ & $1.42 \mathrm{eV}$ & $1.50 \mathrm{eV}$ & $1.81 \mathrm{eV}$ \\
Efficiency & $\eta$ & $\eta$ & $\eta$ & $\eta$ \\
(Voltage) & $\left(\mathrm{V}_{\mathrm{oc}}\right)$ & $\left(\mathrm{V}_{\mathrm{oc}}\right)$ & $\left(\mathrm{V}_{\mathrm{oc}}\right)$ & $\left(\mathrm{V}_{\mathrm{oc}}\right)$ \\
\hline Reported World record cells & $22.1 \%$ & $28.8 \%$ & $19.6 \%$ & $20.8 \%$ \\
& $(0.878 \mathrm{~V})$ & $(1.122 \mathrm{~V})$ & $(0.857 \mathrm{~V})$ & $(1.455 \mathrm{~V})$ \\
Detailed balance substrate cells & $31.5 \%$ & $31.1 \%$ & $30.4 \%$ & $25.7 \%$ \\
& $(1.020 \mathrm{~V})$ & $(1.091 \mathrm{~V})$ & $(1.174 \mathrm{~V})$ & $(1.454 \mathrm{~V})$ \\
Detailed balance mirror cells & $33.7 \%$ & $33.2 \%$ & $32.1 \%$ & $27.0 \%$ \\
& $(1.085 \mathrm{~V})$ & $(1.159 \mathrm{~V})$ & $(1.234 \mathrm{~V})$ & $(1.524 \mathrm{~V})$ \\
\hline \hline
\end{tabular}

conduction band) and $9.52 \times 10^{18} \mathrm{~cm}^{-3}$, respectively. ${ }^{7}$ Thus, from Eq. (8), $n_{i}=2.36 \times 10^{6} \mathrm{~cm}^{-3}$ at $300 \mathrm{~K}$ noting the exponential dependence on temperature.

If the solar cell is fabricated instead on a parasitically absorbing substrate, then the expression for the radiative recombination coefficient is now given by

$$
B=2 \pi\left(1+n_{r}^{2}\right) n_{i}^{-2} h^{-3} c^{-2} \int_{E_{g}}^{\infty} E^{2} \alpha(E) \exp \left(-E / k_{B} T\right) d E .
$$

Using absorption coefficient data for GaAs (at $300 \mathrm{~K}$ ), ${ }^{5}$ Eqs. (7) and (9) are solved numerically. The calculated values of $B$ for GaAs $(300 \mathrm{~K})$ are $1.26 \times 10^{-11} \mathrm{~cm}^{3} \mathrm{~s}^{-1}$ (mirror cell) and $1.81 \times 10^{-10} \mathrm{~cm}^{3} \mathrm{~s}^{-1}$ (substrate cell). Calculations of $B$ are sensitive to $\alpha(E)$ near the bandgap energy and thus differing sets of measured absorption coefficient data can alter the calculated radiative recombination coefficient. Moreover, $B$ is sensitive to $n_{i}$ which itself is a sensitive function of temperature.

All told, perhaps of even more fundamental interest, prior experimental work on GaAs epitaxial layers confirms that the surrounding media influences the radiative recombination coefficient ${ }^{8}$ as indicated here in the comparison between solar cells with mirrors and absorbing substrates. As a general comment, the radiative recombination coefficient will be greater for GaAs, CdTe, InP, and $\mathrm{Ga}_{0.5} \mathrm{In}_{0.5} \mathrm{P}$ substrate cells in comparison to their mirror cell counterparts by a factor of $\left(1+n_{r}^{2}\right)$ as shown in Eq. (9).

\section{LIFETIME}

\section{A. Radiative lifetime}

The minority electron radiative lifetime ${ }^{9}$ in the $p$-type base layer of a solar cell is given by

$$
\tau_{\text {rad }}=\left[B \cdot\left(p_{0}+n_{0}+n_{p h}\right)\right]^{-1},
$$

where $p_{0}$ is the equilibrium hole concentration, $n_{0}=n_{i}^{2} / p_{0}$, and $n_{p h}$ is the photogenerated electron concentration. Each absorbed photon from the Sun is assumed to photogenerate one electron and one hole; therefore, $n_{p h}=p_{p h}$ where $p_{p h}$ is the photogenerated hole concentration.

Before proceeding, it is important to divert briefly and note here that the photogenerated carrier concentrations $\left(n_{p h}, p_{p h}\right)$ at steady state and open circuit account for the carriers generated from absorption of solar radiation as well as the carriers generated from absorption of internal luminescent radiation. The luminescent radiation occurs when photogenerated electrons that subsequently have relaxed to what is known as the conduction band minimum (i.e., the $\Gamma$ valley in GaAs) recombine radiatively with available photogenerated holes that have subsequently relaxed to what is known as the valence band maximum thus emitting photons with energy approximately equal to the bandgap of the semiconductor. The time duration of the carrier relaxation process within the band is several orders of magnitude shorter than the carrier radiative (band to band) lifetime, thus ensuring that the radiative recombination process is dominated by carriers that have relaxed to the fundamental band edges. If the luminescent photons do not escape from the solar cell then they may also be absorbed. This normal process of luminescent radiation emission and absorption in semiconductors was discussed at least as early as $1957 .{ }^{10}$ Luminescent radiation may be emitted in any direction due to momentum randomization and thus on average half of the emitted luminescent radiation is directed toward the rear of the solar cell. In the case of a solar cell fabricated on a parasitically absorbing substrate, the emitted luminescent radiation that is absorbed in the substrate results in an increase in entropy ${ }^{3}$ that otherwise would not occur in solar cells with perfect mirrors in which the rearward emitted luminescent radiation is reflected toward the front of the cell where it either escapes according to Snell's Law or is totally internally reflected. The increased entropy is a degrading factor that causes a reduction to the open circuit voltage of $-k_{B} T e^{-1} \ln (1$ $+n_{r}^{2}$ ) as shown previously in Eq. (2).

Now, the goal is to determine $n_{p h}$ when there is limited cell information, for instance, when $V_{\mathrm{oc}}$ and the doping concentration are unknown. In order to accomplish this, an intrinsic semiconductor is invoked, and the photogenerated carrier concentration is assumed to be a non-degenerate ideal gas. When the semiconductor is irradiated by sunlight, the single Fermi level that existed in the dark at thermal equilibrium prior to illumination is instead expressed under steady-state conditions as two separate quasi-Fermi level$\mathrm{s}$ - one level for the relaxed population of electrons and the other level for the relaxed population of holes. These quasi-Fermi levels are expressed as

$$
\begin{aligned}
& E_{F n}=E_{i}+k_{B} T \ln \left(n / n_{i}\right) \\
& E_{F p}=E_{i}-k_{B} T \ln \left(p / n_{i}\right),
\end{aligned}
$$

where $E_{i}$ is the intrinsic Fermi level and the electron concentration $n$ and hole concentration $p$ are

$$
\begin{aligned}
& n=n_{i}+n_{p h} \\
& p=p_{i}+p_{p h} .
\end{aligned}
$$

In an intrinsic semiconductor $n_{i}=p_{i}$ and since $n_{p h}=p_{p h}$ then $n=p$. The relationship between carrier concentration product $n p$ and quasi-Fermi level separation $E_{F n}-E_{F p}$, where $E_{F n}-E_{F p}=e V_{o c}$, is given by 


$$
n p=n_{i}^{2} \exp \left(e V_{o c} / k_{B} T\right) .
$$

Substituting $V_{o c}$ values calculated from Eqs. (1) and (2) into Eq. (13) gives, for GaAs, $n=p=1.5 \times 10^{16} \mathrm{~cm}^{-3}$ (mirror cell) versus $4.0 \times 10^{15} \mathrm{~cm}^{-3}$ (substrate cell). Meanwhile, for GaAs, $n_{i}=2.36 \times 10^{6} \mathrm{~cm}^{-3}$ as calculated earlier. Therefore, since $n_{i} \ll n(p)$, it is clear from Eq. (12) that $n \approx n_{p h}$ and $p \approx p_{p h}$. This approximation is valid for the solar cells discussed in this paper (InP, GaAs, CdTe, and $\left.\mathrm{Ga}_{0.5} \mathrm{In}_{0.5} \mathrm{P}\right)$. From Eq. (10), $\tau_{\text {rad }}$ may be determined for GaAs mirror and substrate cells as a function of the base layer acceptor concentration $N_{\mathrm{a}}$ as shown in Fig. 2 where $N_{\mathrm{a}} \gg n_{i}$, and the acceptors are assumed to be fully ionized at room temperature such that $p_{0} \approx N_{\mathrm{a}}$. This provides a guide for understanding the maximum radiative lifetime in GaAs cells when typical base doping is considered. Note here that the calculations of radiative lifetime are made at $300 \mathrm{~K}$ whereas solar cells are officially certified at $298 \mathrm{~K}$. In the field, however, it is likely that the solar cell will operate at $300 \mathrm{~K}$ or greater with the main point being that radiative lifetime is a function of temperature.

\section{B. Auger lifetime}

In comparison, for a $p$-type base layer the nonradiative Auger recombination lifetime ${ }^{9}$ is given by

$\tau_{\text {Aug }}=\left[C_{p} \cdot\left(p_{0}^{2}+2 p_{0} n_{p h}+n_{p h}^{2}\right)+C_{n} \cdot\left(n_{0}^{2}+2 n_{0} n_{p h}+n_{p h}^{2}\right)\right]^{-1}$,

where $C_{p}\left(4 \times 10^{-30} \mathrm{~cm}^{6} \mathrm{~s}^{-1}\right)$ and $C_{n}\left(1.8 \times 10^{-31} \mathrm{~cm}^{6} \mathrm{~s}^{-1}\right)$ are Auger recombination coefficients for GaAs (at $300 \mathrm{~K}$ ). ${ }^{9}$ From Eq. (14) with $p_{0}=1 \times 10^{17} \mathrm{~cm}^{-3}, \tau_{A u g}=19 \mu \mathrm{s}$ (mirror cell) versus $23 \mu \mathrm{s}$ (substrate cell). Between the choice of radiative and Auger lifetime, it is the radiative lifetime that limits GaAs solar cells. As a general comment, under one Sun AM1.5G conditions at room temperature and under low injection it is anticipated that Auger recombination will not be a limiting factor for $\mathrm{CdTe}$, InP, or $\mathrm{Ga}_{0.5} \mathrm{In}_{0.5} \mathrm{P}$ solar cells but in order to know for sure requires reliable Auger coefficient data for CdTe, InP, and $1.81 \mathrm{eV} \mathrm{Ga}{ }_{0.5} \mathrm{In}_{0.5} \mathrm{P}$.

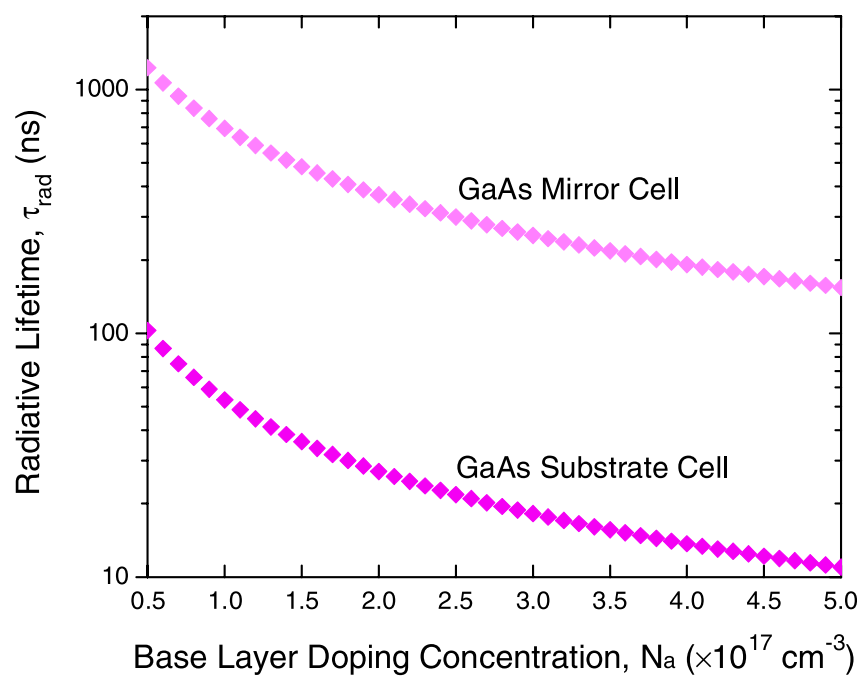

FIG. 2. Radiative lifetime for detailed balance-limiting GaAs mirror and substrate solar cells (AM1.5G spectrum; $300 \mathrm{~K}$ ).

\section{Shockley-Read-Hall lifetime}

Besides Auger recombination, there is also nonradiative SRH recombination which depends on factors that are difficult to characterize including trap concentration $N_{T}$ and capture cross sections $\sigma_{n}$ and $\sigma_{p}$ of the electron and hole traps, respectively. ${ }^{9}$ Variability in the semiconductor layer growth process (growth technique, growth temperature, growth rate, flux ratio, source material purity, and substrate quality) may affect the type and concentration of traps. Molecular beam epitaxy (MBE) is one technique for the epitaxial growth of GaAs. From experiments on MBE-grown GaAs, there are multiple $(\sim 9)$ electron $\operatorname{traps}^{11}$ where $N_{T}$ ranges from $\sim 5 \times 10^{12} \mathrm{~cm}^{-3}$ to $1 \times 10^{14} \mathrm{~cm}^{-3}$. It is assumed that $\mathrm{SRH}$ recombination at surfaces is negligible with near ideal passivation provided by wide bandgap and lattice-matched hetero-interface layers known as window and back surface fields. An example is $\mathrm{Ga}_{0.5} \mathrm{In}_{0.5} \mathrm{P}$ on GaAs where interface recombination velocity - an indication of interfacial SRH recombination-of less than $1.5 \mathrm{~cm} \mathrm{~s}^{-1}$ has been reported. ${ }^{12}$

The SRH lifetime 9 for a $p$-type base layer under low injection $\left(n_{p h}<p_{0}\right)$ is approximated by

$$
\tau_{S R H}=\left(\sigma_{n} v_{t h} N_{T}\right)^{-1}
$$

where $v_{t h}\left(\sim 1 \times 10^{7} \mathrm{~cm} \mathrm{~s}^{-1}\right)$ is thermal velocity ${ }^{7}$ given by

$$
v_{t h}=\sqrt{3 k_{B} T / m^{*}},
$$

and $m^{*}$ is effective mass. As noted by Pierret, ${ }^{7}$ uncertainty about the proper effective mass that should be used in Eq. (16) is typically dealt with by substituting the free electron mass for $m^{*}$. From Eq. (15), $\tau_{S R H}$ for GaAs as a function of $N_{T}$ for $\sigma_{n}=1 \times 10^{-14} \mathrm{~cm}^{2}$ versus $5 \times 10^{-15} \mathrm{~cm}^{2}$ is shown in Fig. 3. Under low injection, SRH lifetime is independent of solar cell configuration (i.e., mirror vs. substrate) because photogenerated carrier concentration is not a contributing variable in Eq. (15). If CdTe, InP, and $\mathrm{Ga}_{0.5} \mathrm{In}_{0.5} \mathrm{P}$ cells have the same trap concentration and capture cross sections as

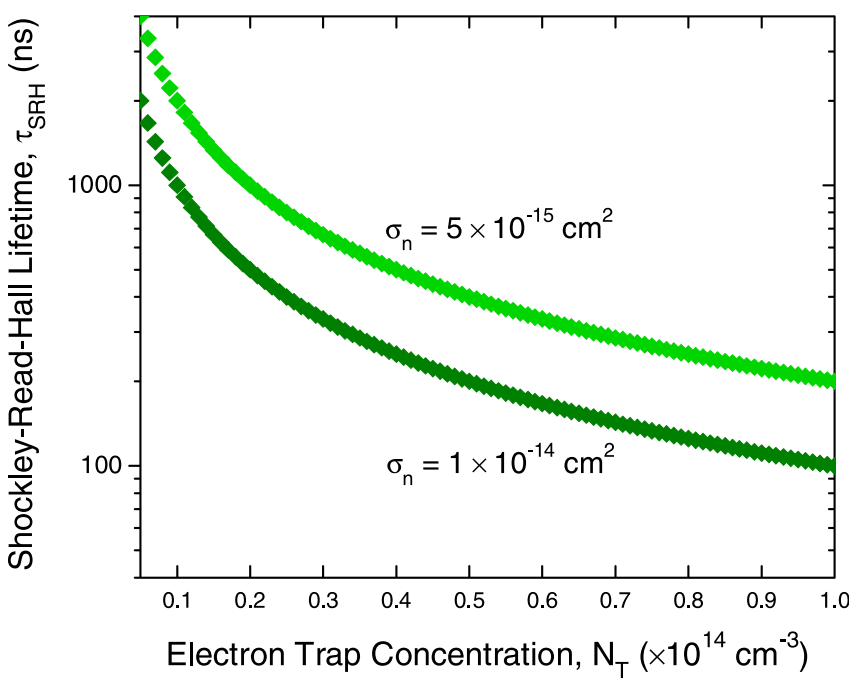

FIG. 3. SRH lifetime in GaAs as a function of electron trap concentration for two different electron capture cross sections. 


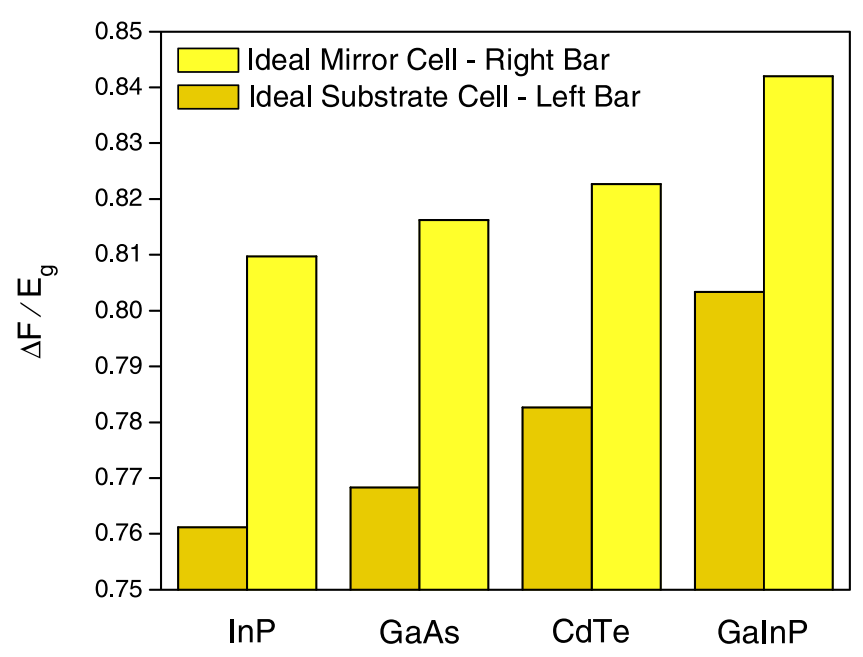

FIG. 4. Limiting figure of merit $\Delta F / E_{g}$ for mirror and substrate solar cells (AM1.5G spectrum; 298 K). Note that the term "ideal" here refers to detailed balance-limiting mirror (substrate) cells.

GaAs, then it is expected at first order that they will have about the same SRH lifetime as the GaAs example presented here.

In order to take advantage of the greater radiative lifetime of mirror solar cells, due diligence at reducing trap density is important for maximizing the SRH lifetime. For GaAs mirror cells (with $p_{0}=1 \times 10^{17} \mathrm{~cm}^{-3}$ ), the lifetime will be about equally limited by radiative and SRH lifetime when $N_{T}$ is $\sim 1 \times 10^{13} \mathrm{~cm}^{-3}$ and $\sigma_{n}=1 \times 10^{-14} \mathrm{~cm}^{2}$. Larger capture cross section and/or trap concentration will then cause the mirror cell to become SRH limited. However, even if the radiative limit can be achieved, other losses that degrade the peak efficiency of the solar cell include $I^{2} R$ loss in the top grid contacts as well as the emitter/window, and also failure to achieve complete photon absorption due to grid contact and busbar shading plus imperfect antireflection coatings that do not have zero reflectivity over the entire portion of the polychromatic solar spectrum containing photons with energy greater than or equal to the bandgap energy of the solar cell.

\section{FREE ENERGY}

The change in free energy $\Delta F$ of the photogenerated carriers is equal to $e V_{o c}{ }^{3}$ The figure of merit $\Delta F / E_{g}$ for detailed balance-limiting InP, GaAs, CdTe, and $\mathrm{Ga}_{0.5} \mathrm{In}_{0.5} \mathrm{P}$ solar cells is shown in Fig. 4. It is apparent that $1.81 \mathrm{eV} \mathrm{Ga}_{0.5} \operatorname{In}_{0.5} \mathrm{P}$ solar cells with a bandgap energy corresponding to visible light outperform the other three solar cells that have a bandgap energy corresponding to the near-IR portion of the spectrum. From the viewpoint of the change in free energy $\Delta F$ "normalized" to each cell's bandgap $E_{g}$, the $\mathrm{Ga}_{0.5} \mathrm{In}_{0.5} \mathrm{P}$ cells in this sense are more effective than the other three solar cells because the high quality near-UV and visible photons are better utilized in the $1.81 \mathrm{eV}$ bandgap cell than in the lower bandgap cells which waste a significant amount of the photogenerated carrier energy via hot carrier relaxation. Stated another way, by plotting $\Delta F / E_{g}$ it is possible to quantify for any given solar cell and its particular bandgap (i.e., its quantum threshold) the fraction of incident solar energy (i.e., absorbed light quanta described by $h v \geq E_{g}$ ) that ultimately results in open circuit voltage.

\section{CONCLUSION}

In summary, first principle physics has been utilized to compare direct bandgap InP, GaAs, CdTe, and $\mathrm{Ga}_{0.5} \mathrm{In}_{0.5} \mathrm{P}$ solar cells with absorbing substrates versus mirrors. The principle of detailed balance may be employed to characterize substrate versus mirror solar cells in terms of not only open circuit voltage and power conversion efficiency but also in terms of radiative recombination coefficient necessary to compute radiative lifetime. Mirror solar cells generate greater voltage and efficiency than their substrate counterparts while also offering longer radiative lifetime. Wider bandgap cells that have a threshold (bandgap energy) corresponding to visible light make better use of the incident high quality near-UV and visible photons than cells with thresholds in the near-IR, even when the open circuit voltage multiplied by the electronic charge is then normalized to the bandgap. These wider bandgap cells, which are of critical importance to the next generation of 4-6 junction solar cells, manage the change in free energy of the photogenerated and then relaxed electrons and holes more optimally when compared to near-IR threshold cells such as InP, GaAs, and CdTe.

\section{ACKNOWLEDGMENTS}

A.P.K. thanks Professor Y.-H. Zhang (Arizona State University) for helpful discussions and the Bisgrove Postdoctoral Scholars program for support. W.P.K. thanks NASA (Award Nos. NCC-1-02038 and NCC-8-236) and Texas Instruments, Inc. (gift award) for partial support. This material is based upon work supported by the National Science Foundation under Grant No. ECCS-1214172.

${ }^{1}$ M. A. Green, K. Emery, Y. Hishikawa, W. Warta, and E. D. Dunlop, Prog. Photovoltaics 21(5), 827 (2013).

${ }^{2}$ W. Shockley and H. J. Queisser, J. Appl. Phys. 32(3), 510 (1961).

${ }^{3}$ C. H. Henry, J. Appl. Phys. 51(8), 4494 (1980).

${ }^{4}$ J. F. Geisz, M. A. Steiner, I. García, S. R. Kurtz, and D. J. Friedman, Appl. Phys. Lett. 103(4), 041118 (2013).

${ }^{5} \mathrm{~S}$. Adachi, Optical Constants of Crystalline and Amorphous Semiconductors (Kluwer Academic Publishers, Boston, 1999).

${ }^{6} \mathrm{~J}$. Nelson, The Physics of Solar Cells (Imperial College Press, London, 2003).

${ }^{7}$ R. F. Pierret, Advanced Semiconductor Fundamentals, 2nd ed. (Prentice Hall, NJ, 2003).

${ }^{8}$ E. Yablonovitch, T. J. Gmitter, and R. Bhat, Phys. Rev. Lett. 61(22), 2546 (1988).

${ }^{9}$ D. K. Schroder, Semiconductor Material and Device Characterization (John Wiley \& Sons, New York, 1990).

${ }^{10}$ W. P. Dumke, Phys. Rev. 105(1), 139 (1957).

${ }^{11}$ D. V. Lang, A. Y. Cho, A. C. Gossard, M. Ilegems, and W. Wiegmann, J. Appl. Phys. 47(6), 2558 (1976).

${ }^{12}$ J. M. Olson, R. K. Ahrenkiel, D. J. Dunlavy, B. Keyes, and A. E. Kibbler, Appl. Phys. Lett. 55(12), 1208 (1989). 